\title{
El tipo de cambio renminbi-dólar y su impacto sobre las exportaciones e Inversión Extranjera Directa en México
} Belem Iliana Vásquez Galán*

El Colegio de la Frontera Norte, Departamento de Estudios Económicos

\section{Cuauhtémoc Calderón Villarreal}

El Colegio de la Frontera Norte, Departamento de Estudios Económicos

(Recibido 15 de Julio 2016, aceptado 04 de Octubre 2016)

\begin{abstract}
Resumen
Este es un análisis del efecto que tiene una fluctuación del tipo de cambio renminbi-dólar sobre las exportaciones mexicanas dirigidas al mercado estadounidense y sobre la inversión extranjera directa procedente de ese país. Las estimaciones de un vector de corrección de error, con datos de 1995 a 2015, indican que una depreciación del renminbi reduce el crecimiento de las exportaciones mexicanas en 2.01 por ciento, pero no tiene efecto sobre la IED. Esto implica que, al ser competidores cercanos con China, el sector exportador mexicano es vulnerable a la política monetaria china que favorece la depreciación de su moneda y, que con ello contribuye a desplazar las exportaciones mexicanas. Los resultados ofrecen apoyo para entender una de las causas por las cuales México ha perdido participación en el mercado estadounidense mientras China la ha ganado. En comparación con otros estudios, nuestros resultados, con un periodo más amplio, nos permiten concluir que la diversificación de las exportaciones incrementa la sensibilidad a una depreciación del renminbi por ello, es recomendable el fomento de bienes de exportación con menor elasticidad.
\end{abstract}

Clasificación JEL: C32, E5, F10, F31, F51.

Palabras clave: Tipo de cambio, Renminbi, Exportaciones, Inversión Extranjera Directa.

\section{The Renminbi Exchange Rate and its Impact on Mexicos' exports and Foreign Direct Investment.}

\section{Abstract}

This is an analysis of the effect of a fluctuation of the renminbi-dollar exchange rate on Mexican exports directed to the US market and on foreign direct investment coming from the United States. Estimates of an error correction vector with data from 1995 to 2015 indicate that a depreciation of the renminbi reduces the growth of Mexican exports by 2.01 percent but has no effect on FDI. Implying that by being close competitors with China, the Mexican export sector is vulnerable to Chinese monetary policy which favors the depreciation of its currency and, with that, contributes to the displacement of Mexican exports. The results of our article offer empirical evidence to be able to understand why Mexico has lost its share in the US market while China has gained even more. Compared with other studies, our results, with a more ample period, allow us to conclude that the diversification of exports increases

* El Colegio de la Frontera Norte, A. C. Km. 18.5 Carretera Escénica Tijuana - Ensenada San Antonio del Mar Tijuana, Baja California, México, C.P. 22560. Tel. 6316300 ext. 3418. Correo Electrónico:calderon@colef.mx 
the sensitivity to a depreciation of the renminbi thus, it is advisable to promote the export of goods with less elasticity.

JEL Classification: C32, E5, F10, F31, F51.

Key Words: Exchange rate, Renminbi, Exports, Foreign Direct Investment.

\section{Introducción}

En agosto de 2010, países como Alemania y Japón se unieron a los Estados Unidos de América (EUA) para presionar a China y que ésta permitiera que su moneda el renminbi ${ }^{1}$ se apreciara. La razón es que desde que la determinación del tipo de cambio entró a un régimen de flotación controlada en 2005, el gobierno chino estuvo manipulando su valor ${ }^{2}$ con el objeto de mantener la moneda subvaluada y así generar ganar competencia desleal con los países que se estaban recuperando de la crisis de 2008 (Shannon, 2010).

México como competidor cercano de China en el mercado de EUA no es ajeno a la problemática que representa la sobrevaluación del tipo de cambio, ya que éste afecta directamente no sólo la balanza comercial, sino también la competitividad de las exportaciones mexicanas y su capacidad de atraer recursos financieros internacionales. Muchas de las exportaciones chinas a EUA son productos intensivos en mano de obra, lo que le da ventaja comparativa frente a otros países. Por ejemplo, en el caso de México, los principales productos de exportación provienen de la industria manufacturera en partidas similares a los productos chinos. Una posible depreciación de la moneda china implica que, asumiendo que se trata de productos homogéneos, la demanda de exportaciones mexicanas frente a las chinas se reducirá. La pérdida (o ganancia) de competitividad de las exportaciones mexicanas, frente a las exportaciones chinas, se mide por el desplazamiento en contra (o a favor) de los productos mexicanos cuando el renminbi se deprecia (o aprecia) frente al dólar. Esto implica que, los productos mexicanos que son cercanos competidores con los productos chinos en el mercado de EUA, por ejemplo las manufacturas, serán más vulnerables a las fluctuaciones del tipo de cambio renminbi-dólar.

El análisis del efecto que ha tenido la mayor participación de China en el comercio mundial y sobre todo en EUA ha sido abordado desde diferentes perspectivas. En el caso específico de cómo los productos chinos han desplazado a ciertos productos mexicanos en el mercado de importaciones de EUA, Watkins (2015) afirma que, a pesar de que entre 2000 y 2013 las importaciones de productos chinos en EUA aumentaron $338 \%$ y los productos mexicanos $106 \%$, México mantiene una posición de ventaja debido a que sus exportaciones contienen un alto índice de peso-valor, cuentan con protección de propiedad intelectual y se concentran en productos intensivos en tecnología como los de la industria automotriz, aeroespacial y equipo médico. Un aspecto importante

\footnotetext{
1 Aunque renminbi y yuan se usan indistintamente, en realidad se refieren a dos conceptos que hay que diferenciar. El nombre oficial de la moneda China es "renminbi" que se traduce del chino como "la moneda de la gente". El yuan es la unidad de medida del renminbi, mientras que jiao y fen son las medidas fraccionarias (The Peoples Bank of China, 2015).

2 La política cambiaria del gobierno chino ha sido un instrumento central para expandir sus exportaciones, que en las últimas décadas han crecido de manera extraordinaria. Desde 1985, el tipo de cambio real efectivo chino se ha depreciado más del $100 \%$.
} 
mencionado por Watkins, es que el sector manufacturero mexicano debe parte de su competitividad a la mayor alianza que tienen con empresas asociadas de EUA a través de plantas ensambladoras ubicadas en territorio mexicano. En un análisis detallado, Dussel y Gallagher (2013) calcularon la participación de las exportaciones chinas y mexicanas en el mercado estadounidense, entre 2000 y 2009, para identificar productos mexicanos bajo amenaza directa con China. Encontraron que $52 \%$ de las manufacturas mexicanas perdieron participación en el mercado estadounidense, mientras que las manufacturas chinas la ganaron, lo que implica un desplazamiento o pérdida de competitividad para México. El porcentaje de exportaciones mexicanas en general que se identificaron bajo amenaza directa fue de $36 \%$.

Parte de la competitividad que adquirieron las exportaciones chinas a partir de su integración a la Organización Mundial del Comercio (OMC) en 2001, se debió a la política monetaria del Banco Central que mantenía depreciado el tipo de cambio renminbi y con ello abarataba las exportaciones. La manipulación del valor de la moneda china fue objeto de reclamos de varios países que veían incrementar sus déficits de cuenta corriente. En julio de 2005, el Banco Central de China introdujo un régimen flexible con una banda de flotación que permitió un lento descenso del tipo de cambio del renminbi y que hasta 2013 tenía una tasa de apreciación acumulada de 36\%, (Perrotini \& Liu Sun, 2015).

En este contexto, el objetivo de este trabajo es determinar si la fluctuación del renminbi frente al dólar ha tenido un impacto negativo sobre la economía mexicana en dos vertientes: en la competitividad de sus exportaciones a EUA, y en la inversión extranjera directa recibida de ese país. Se asume la postura de que una depreciación del renminbi tiene un efecto negativo sobre el crecimiento de las exportaciones de México al ser competidor cercano en productos similares, y en los flujos de Inversión Extranjera Directa (IED) al asumir la posible desviación de inversión ante al abaratamiento de los costos de operación en China. En este análisis se utilizan herramientas de series de tiempo como cointegración y vectores de corrección de errores.

\section{Algunos aspectos teóricos y contextuales}

De acuerdo con la teoría del comercio internacional estándar hay dos razones por las cuales los países se especializan en la producción de bienes e intercambian sus productos. La primera es porque los países se diferencian en recursos productivos y nivel tecnológico, de manera que se especializan en la producción en la cual tienen una ventaja comparativa. Y la segunda es porque las economías de escala hacen que sea más ventajoso que cada país se especialice en un número limitado de bienes y servicios (Krugman \& Obstfeld, 2006). De esta forma, los países ganan en el comercio internacional al ampliar la frontera de posibilidades de producción, y la especialización permite que los recursos se distribuyan hacia los sectores que pagan los salarios más altos. El modelo de ventaja comparativa se basa en el supuesto de rendimientos constantes a escala, existe abundante literatura que explica las variantes teóricas que se fueron adicionando al modelo básico de David Ricardo (Bhagwati, Panagariya, \& Srinivasan, 1998).

Conforme se ha ido desarrollando el mercado internacional de comercio, también se han generado nuevas formas de producción que han dado lugar a una nueva interpretación del comercio. En la nueva teoría del comercio 
internacional se elimina el supuesto de rendimientos constantes a escala pues la evidencia empírica demuestra que muchas industrias se caracterizan por tener economías de escala, lo que ocasiona que la producción sea más eficiente conforme la escala de operación aumenta. También se reconoce que mucho del comercio internacional se da entre países con recursos productivos y niveles de productividad similares (conocido como comercio intra-industrial) y que mucha de la producción mundial es generada por empresas multinacionales (Krugman, 1980).

Para la nueva teoría del comercio, la competencia imperfecta es una consecuencia necesaria de las economías a escala, ya que las empresas que logran producir a escalas superiores son capaces de fijar los precios y tener mayor poder de mercado. En competencia imperfecta las empresas pueden fijar un precio diferente si el bien es exportado o si se vende en el mercado nacional. Esta práctica de discriminación de precios es conocida como "dumping" en el comercio internacional. El dumping, que es promovido por empresas o naciones, es usado como estrategia para maximizar los beneficios ya que los precios de los bienes exportados se abaratan de manera artificial, pero dado que es una práctica desleal se encuentra sujeta a sanciones comerciales (OMC, 1994). Algunas de las medidas más comunes para alterar los precios de los bienes exportados son los subsidios a la producción, la exención de impuestos y el mantenimiento del valor de la moneda subvaluada con el fin de hacer más baratos los bienes en el extranjero. En este sentido el papel de los gobiernos nacionales es clave para entender su participación como agente promotor de estas prácticas, que aunque distorsionan los precios de intercambio, les permite fortalecer y aumentar la competitividad de su sector exportador.

México como competidor con China en el mercado de EUA ha sido desplazado ya como segundo socio comercial. En 2003 por primera vez China se convierte en la segunda fuente de importaciones de EUA al vender exportaciones valoradas en $\$ 152,436$ millones de dólares frente a $\$ 138,160$ millones de dólares de México. En la gráfica 1 se puede observar que no sólo China superó a México desde 2003 sino que la brecha entre ambas naciones parece aumentar a lo largo de los últimos años. Mientras que en 1990 México exportaba a EUA el doble del valor de las exportaciones chinas, en 2015 esa relación fue inversa.

Gráfica 1. Exportaciones de bienes de México y China a los EUA

(en millones de dólares)

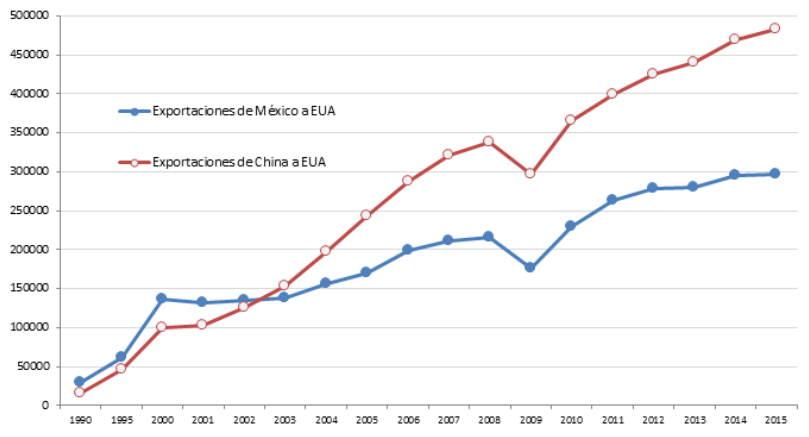

Fuente: elaboración propia con datos de US Census Bureau (2016). 
China preparó estratégicamente su adhesión a la OMC en diciembre de 2001, pues implicó mejorar la calidad de los productos ofrecidos en el mercado internacional y aumentar el nivel tecnológico incorporado en sus exportaciones, además de otros aspectos requeridos (WTO, 2001). De acuerdo a la CEPAL, mientras en 1980 alrededor de $22 \%$ de las exportaciones chinas totales tenían nivel tecnológico medio y alto, en México el porcentaje era de 47\%. Para 2002, China incrementó el porcentaje a $65 \%$ de los productos exportados, mientras que México redujo el porcentaje a 42\% (Dussel, 2009).

A partir de la entrada en vigor del Tratado de Libre Comercio de América del Norte (TLCAN) en 1994, el crecimiento de las exportaciones de manufacturas mexicanas se acrecentó, y por algunos años mantuvo este dinamismo, el sector exportador (no petrolero) se concentraba en las manufacturas y en específico en la industria maquiladora. En 2001 con los efectos de la desaceleración del crecimiento de la economía estadounidense, las exportaciones entran en un periodo de caída (ver gráfica 2). Muchas empresas tuvieron que reducir sus niveles de producción ante la menor demanda en EUA y también disminuyeron los flujos de inversión directa a México. La siguiente caída en las exportaciones ocurrió en 2009 por los efectos de la crisis financiera de EUA y la subsecuente reducción en la demanda de productos importados.

Gráfica 2. Exportaciones totales de México a EUA de 1993:1 a 2015:12

(en millones de dólares)

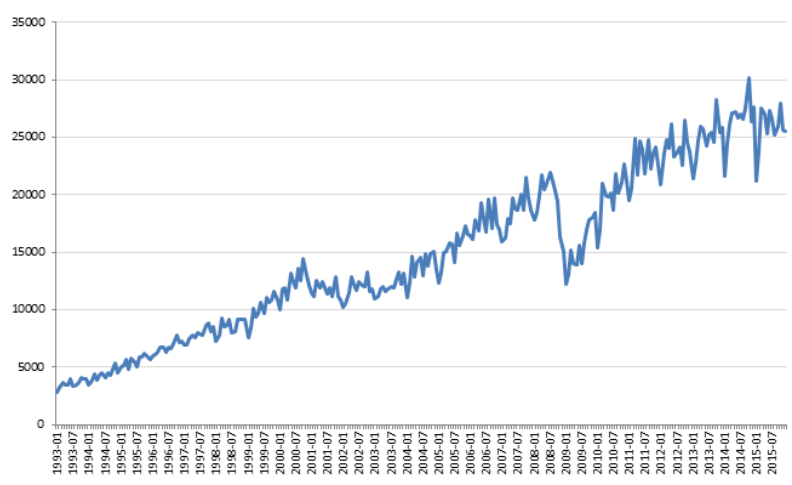

Fuente: Elaboración propia con datos de Banxico (2016).

Carrillo y Plascencia (2007) sostienen que desde que China adoptó el modelo maquilador en 1978, se convirtió en el principal competidor de México al pagar salarios tres veces más bajos que en México. Esto permitió que China se convirtiera en el principal productor mundial en calzado, textiles y juguetes. En la producción de televisores y monitores, donde México tenía $65.6 \%$ del mercado estadounidense en 1995, con la entrada de China a la OMC ésta aumentó su participación de $2.6 \%$ en 1995 a 8.5\% en 2002. Chávez y Marín (2007) realizaron un análisis de la competencia de ambos países en el mercado estadounidense, utilizando datos de 1996 a 2004, y encontraron qué, conforme pasan los años, aumenta el número de productos con los que China compite en EUA. Por el otro lado, en esta competencia México ha perdido participación en las importaciones 
totales de EUA que se cuantifican como pérdidas equivalentes a 2,912 millones de dólares en 2002, a 5,662 millones en 2003 y 3,553 millones en 2004.

La identificación de los productos mexicanos que han perdido participación, mientras que China la ha ganado, nos permite indagar si esto ha fomentado la especialización en bienes donde México mantiene una clara ventaja frente a China a pesar de la subvaluación del renminbi. En el cuadro 1 se presentan veinte de los productos más importados (en términos de valor) por EUA en 2008 y 2015, ordenados por importancia. Los autos de pasajeros (clasificados en la partida 8703 del sistema armonizado) fueron el principal producto importado por EUA en ambos años de referencia. 10.9\% del valor de las importaciones de autos importados provinieron de México en 2008 y $14.2 \%$ en 2015. En la misma rama automotriz, México mantuvo y mejoró su posición como principal proveedor de camiones y autopartes (partidas 8708 y 8704 ) en ese periodo. Aunque China no representa ningún tipo de competencia para México en la industria automotriz, si ha ganado competencia en otras partidas como la 8517, que corresponde a aparatos de telefonía, donde México perdió 5.2 puntos porcentuales (pp) mientras que China ganó $23.4 \mathrm{pp}$. Esto representa para México una pérdida de competitividad valorada en 5,374 millones de dólares.

Cuadro 1. Valor de los principales productos (no petroleros) importados por

EUA y participación de México y China en el total. Años 2008 a 2015.

\begin{tabular}{|c|c|c|c|c|c|c|c|c|c|c|}
\hline \multirow[t]{2}{*}{$\begin{array}{c}\text { Partida } \\
\mathrm{HS}\end{array}$} & \multirow[t]{2}{*}{ escripción } & \multicolumn{2}{|c|}{ (Millones USD) } & \multicolumn{2}{|c|}{ México (\%) } & \multicolumn{2}{|c|}{ China (\%) } & \multicolumn{2}{|c|}{$\begin{array}{l}\text { Ganancia o pérdida } \\
\text { en puntos porc. } \\
(2008-2015)\end{array}$} & \multirow{3}{*}{$\begin{array}{r}\begin{array}{c}\text { Ganancia o } \\
\text { pérdida } \\
\text { (millones } \\
\text { USD) }\end{array} \\
\text { México } \\
5434.8\end{array}$} \\
\hline & & 2008 & 2015 & 2008 & 2015 & 2008 & 2015 & México & China & \\
\hline 8703 & Autos de pasjeros & 127935 & 169139 & 10.9 & 14.2 & 0.1 & 0.2 & 3.2 & 0.1 & \\
\hline 8517 & Aparatos de telefonia & 61214 & 102369 & 16.8 & 11.6 & 37.6 & 61.0 & -5.2 & 23.4 & -5373.6 \\
\hline 8471 & Computadoras & 57511 & 81385 & 9.5 & 20.6 & 53.0 & 63.4 & 11.1 & 10.5 & 9025.3 \\
\hline 8708 & Partes y accesorios de autos & 41865 & 66480 & 22.6 & 32.2 & 9.4 & 14.7 & 9.6 & 5.3 & 6363.2 \\
\hline 8528 & Receptos de TV incluyendo proyectores & 40343 & 26914 & 45.5 & 45.8 & 43.1 & 42.2 & 0.3 & -0.9 & 93.9 \\
\hline 8443 & \begin{tabular}{|l|} 
Máquinas impresoras \\
\end{tabular} & 21059 & 18256 & 2.8 & 3.9 & 37.1 & 42.6 & 1.1 & 5.5 & 193.7 \\
\hline 8542 & \begin{tabular}{|l|} 
Circuitos eléctricos integrados \\
\end{tabular} & 19773 & 28810 & 0.8 & 0.6 & 5.7 & 6.9 & -0.2 & 1.3 & -58.4 \\
\hline 8473 & $\begin{array}{l}\text { Accesorios de computadoras y máquinas de } \\
\text { oficina }\end{array}$ & 19275 & 17666 & 1.7 & 2.3 & 47.8 & 63.9 & 0.6 & 16.0 & 104.5 \\
\hline 9403 & Otros muebles y partes & 17856 & 22236 & 3.6 & 5.0 & 49.2 & 47.2 & 1.4 & -2.0 & 320.7 \\
\hline 8411 & \begin{tabular}{|l|l} 
Turbo-jets, turbo-propeler y otras turbinas \\
\end{tabular} & 16298 & 22247 & 4.3 & 4.4 & 2.1 & 2.5 & 0.1 & 0.4 & 32.2 \\
\hline 9401 & Sillones (p/dentistas \& barberos sillas, etc) & 14414 & 23671 & 22.1 & 30.4 & 48.2 & 43.9 & 8.3 & -4.2 & 1964.9 \\
\hline 6110 & \begin{tabular}{|l} 
Jerseys, cárdigans, etc. \\
\end{tabular} & 14294 & 15441 & 2.8 & 1.3 & 31.0 & 37.7 & -1.5 & 6.7 & -229.7 \\
\hline 9018 & Instrumentos médicos y de veterinaria & 14280 & 20547 & 23.6 & 25.5 & 4.3 & 7.3 & 1.9 & 3.0 & 387.9 \\
\hline 8525 & $\begin{array}{l}\text { Cám aras de TV, de transmisión y radio- } \\
\text { telefonia }\end{array}$ & 13668 & 10572 & 4.3 & 2.6 & 40.0 & 44.4 & -1.7 & 4.4 & -183.0 \\
\hline 9504 & Articulos de diversión juegos y boliches & 13066 & 6443 & 0.7 & 2.1 & 90.2 & 88.8 & 1.3 & -1.4 & 86.6 \\
\hline 8544 & Cables insulados & 12600 & 19904 & 47.0 & 49.9 & 20.8 & 22.4 & 2.9 & 1.6 & 581.0 \\
\hline 6204 & Ropapara mujer & 12411 & 10504 & 4.0 & 2.3 & 38.6 & 40.9 & -1.7 & 2.3 & -180.5 \\
\hline 6403 & Zapatos de piel y sintéticos & 11904 & 13369 & 1.6 & 2.9 & 67.0 & 53.6 & 1.3 & -13.4 & 167.4 \\
\hline 8504 & Transformadores eléctricos y converti dores & 11744 & 14541 & 19.0 & 16.2 & 33.5 & 40.7 & -2.9 & 7.2 & -416.3 \\
\hline 8704 & Camiones para transporte de mercancia & 11726 & 23541 & 63.1 & 83.6 & 0.1 & 0.0 & 20.5 & -0.1 & 4825.6 \\
\hline
\end{tabular}

Fuente: Elaboración propia con datos de Comtrade (2016) Nota: la ganancia o pérdida en millones de USD se calcula multiplicando el valor de las importaciones de EUA de 2015 por los puntos porcentuales ganados o perdidos entre cien. 
De los veinte productos importados con mayor valor, en realidad México sólo tuvo pérdida de competitividad en seis de ellos. Además de los aparatos de telefonía, fueron: los transformadores eléctricos (8504), jersey y ropa de abrigo (6110), ropa para mujer (6204), cámaras de TV y radio telefonía (8525) y circuitos eléctricos (8542). Como se puede ver, una de las industrias perdedoras mexicanas ha sido la de confección de ropa. Gracia-Hernández (2013) realizó un análisis detallado de esta industria y encontró que de treinta ramas de la cadena hilo-textil-confección que eran líderes en 2000, sólo cinco mantuvieron un crecimiento positivo de sus exportaciones en 2008. En general, estos datos del cuadro 1 indican, en algunos casos, China ha ganado participación en el mercado estadounidense pero no a costa de la participación de México. Por ejemplo, la mayor ganancia en valor para México fue en exportación de computadoras (8471), donde aumentó su participación 11 pp que equivalen a 9025 millones de dólares, sin embargo China también aumentó su participación en 10.5 pp. Existen pocos estudios formales que midan el efecto de la moneda china sobre la economía mexicana, Cuevas (2014) estimó una función de exportaciones, con datos 2007 a 2012 , y encontró que por cada punto porcentual que se deprecia el renminbi las exportaciones manufactureras mexicanas se reducen en 1.08 puntos porcentuales. También Cuevas (2016) encontró que una depreciación real del renminbi de un punto porcentual reduce el crecimiento de competitividad internacional de las exportaciones manufactureras en 0.32 puntos porcentuales.

En los noventa, el tipo de cambio chino ajustado al dólar creó presiones inflacionarias que ocasionaron que el Banco de China expandiera la base monetaria a través de la compra de divisas. En la gráfica 3, se muestra la trayectoria del tipo de cambio nominal renminbi-dólar de 1991 hasta 2015. Antes de 1994, el valor del renminbi fluctuaba de acuerdo a la oferta y demanda de la moneda, por lo que la moneda se depreció rápidamente frente al dólar entre 1990 y 1993. Es en enero de 1994 cuando sufrió una devaluación de $49.6 \%$, al pasar de 5.82 a 8.72 yuan por dólar. A partir de entonces, la política monetaria del Banco Popular Chino decidió el valor de la moneda al valor del dólar americano, lo que permitió que por muchos años se mantuviera el tipo de cambio en alrededor de 8.30 yuanes por dólar. En la gráfica 3 claramente se observa que a partir de enero de 1995 y hasta mayo de 2005, el tipo de cambio se mantuvo casi sin cambios, al pasar de 8.46 a 8.27 yuanes por dólar. Es decir, la depreciación promedio anual fue tan sólo de $0.22 \%$, por lo que en diez años la depreciación acumulada del renminbi fue de $2.26 \%$. 
Gráfica 3. Tipo de cambio renminbi-dólar. Periodo 1991:1 a 2015:12

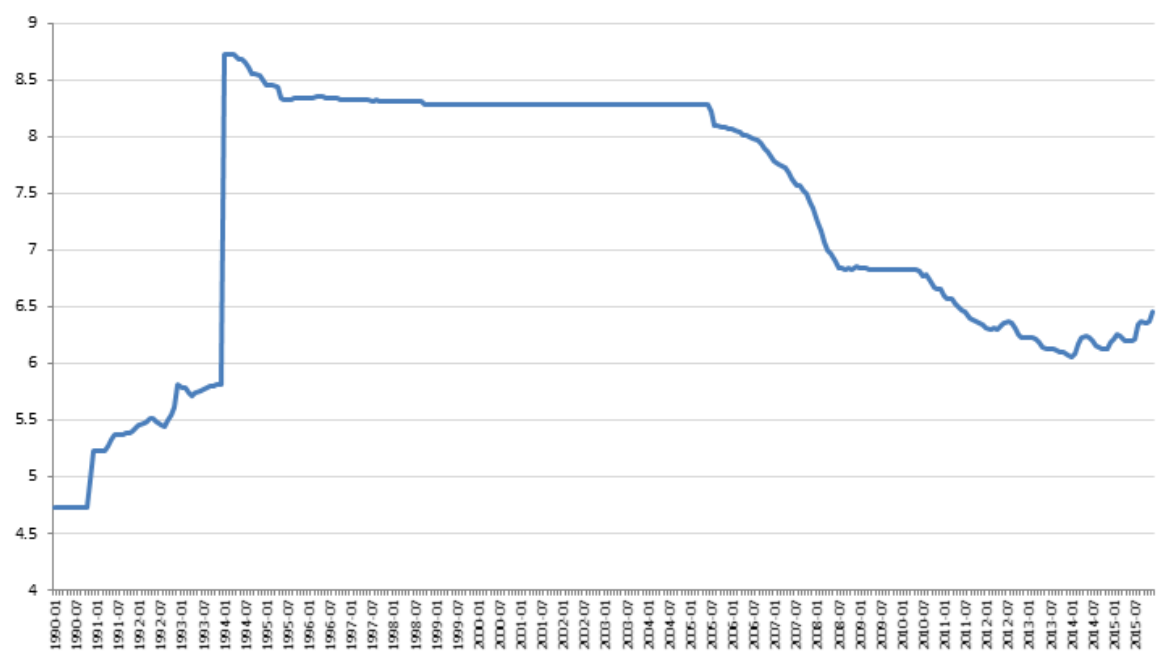

Fuente: Elaboración propia con datos de la Federal Reserve System (2016).

Ante la presión internacional que se generó alrededor de 2005 por la persistencia de las autoridades monetarias chinas de mantener el tipo de cambio fijo (Frankel \& Wei, 2007), y también para disminuir el costo de sus importaciones, pues tenían una fuerte demanda de productos primarios, en julio de 2005 el Banco Popular de China introdujo una banda de flotación de $-/+3 \%$ y fijó el tipo de cambio del renminbi contra una canasta de monedas de países con los que se tenían fuertes vínculos comerciales y financieros (Xiaochuan, 2005). Esta medida de política monetaria permitió inmediatamente la apreciación del renminbi en $1.5 \%$ para agosto de ese año y siguió la misma tendencia los siguientes meses, alcanzando la mayor apreciación anual en 2008 con $8.6 \%$ (ver gráfica 4). La apreciación de la moneda no tuvo la rapidez que esperaban los mercados internacionales, pues lo que se requería era que la autoridad monetaria dejara al libre mercado la cotización de la moneda, principalmente porque a pesar de la banda de flotación introducida, China continuó incrementando el superávit de cuenta corriente como porcentaje del PIB para 2005, 2006 y 2007 (Corden, 2009). En realidad se consideraba que la banda de flotación no permitía el ajuste a un nivel de equilibrio del tipo de cambio. Autores como Goldstein y Lardy (2008) sostienen que para reducir el superávit de cuenta corriente de China se requería una apreciación del tipo de cambio real efectivo de 17 a $30 \%$ y para eliminar el superávit la apreciación tendría que ser de 30 a $55 \%$. Muchos estudios han ofrecido evidencia de que el tipo de cambio continua sobrevaluado, pero no coinciden en el porcentaje en que se debería apreciar el renminbi para alcanzar un nivel de equilibrio (ver Cline y Williamson (2008) para una revisión de la literatura). 
Gráfica 4. Fluctuación promedio anual del tipo de cambio renminbi-dólar (variación porcentual)

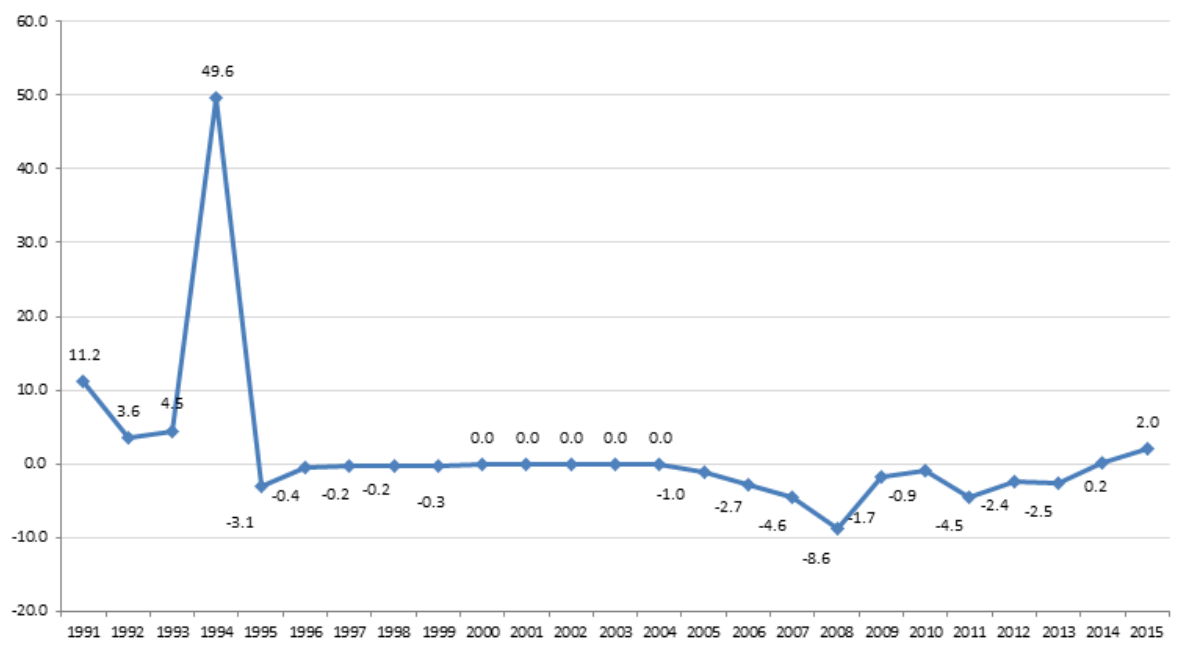

Fuente: Elaboración propia con datos de la Reserva Federal de Estados Unidos (2016).

Debido a que, durante este período de subvaluación del renminbi, la industria manufacturera mexicana tuvo una fuerte caída en el empleo y en la inversión (Mendoza, 2010), surge la pregunta si la pérdida de competitividad exportadora está o no relacionada con la fluctuación de la moneda china, ceteris paribus. Para investigar esta problemática que nos permita entender qué tan expuesta está la economía a través de sus exportaciones e IED a la política monetaria del gobierno chino, se hizo un análisis dinámico para determinar si la depreciación del renminbi ha ocasionado que las exportaciones mexicanas no petroleras pierdan competitividad en el mercado estadounidense y México reduzca en capacidad de atraer IED procedente de ese país. Para ello hay que hacer tres supuestos. Primero, que la mayor parte de las exportaciones se dirigen al mercado de EUA, segundo que la mayor parte de IED proviene de este país y tercero que México y China compiten en EUA con productos similares.

\section{Metodología}

Para determinar la relación que existe entre la economía mexicana y la fluctuación de la moneda china se realizó un análisis dinámico de la relación que existe entre la IED y las exportaciones con respecto al valor del renminbi. En términos generales:

$$
\begin{aligned}
& E X=f(Y u a n, I E D, I P U S) \\
& I E D=f(Y \text { Yan }, E X, I P U S)
\end{aligned}
$$

Donde, $E X$ son las exportaciones no petroleras de México a EUA, IED es el flujo de inversión extranjera directa de EUA en México, Yuan, es el tipo de cambio renminbi-dólar, e IPUS es una variable de control que representa al índice de producción industrial de EUA (año base es 2010). Para el propósito 
de estimar las expresiones (1) y (2) se convirtieron las variables a logaritmos naturales. Por lo tanto las ecuaciones quedan expresadas como elasticidades:

$$
\begin{aligned}
& \log E X=\beta_{0}+\beta_{1} \log Y U A N+\beta_{2} \log I P U S+\beta_{3} \log I E D+\varepsilon_{t} \\
& \operatorname{LogIED}=\beta_{0}+\beta_{1} \log Y U A N+\beta_{2} \log I P U S+\beta_{3} \log E X+\varepsilon_{t}
\end{aligned}
$$

Donde $\log$ se refiere al logaritmo natural de la variable, las $\beta$ son los coeficientes y $\varepsilon$ son los errores. Nuestras expectativas a priori son que: $\beta_{1}<0, \beta_{2}>0, \mathrm{y}$ $\beta_{3}>0$.

Como se puede observar en las expresiones (3) y (4) tanto la variable $Y U A N$ como IPUS son exógenas. A fin de considerar la dinámica del modelo, ambas ecuaciones se estimaron mediante vectores autoregresivos (VAR):

$$
y_{t}=\Pi_{0}+\Pi_{1} y_{t-1}+\Pi_{2} y_{t-2}+\ldots+\Pi_{p} y_{t-p}+\Omega_{1} X_{1 t}+\Omega_{2} X_{2 t}+\varepsilon_{t}
$$

donde $y_{t}$ es un vector $(2 \times 1)$ de las variables endógenas, $X_{m}$ es un vector $(2 \times 1)$ de variables exógenas con $m=1,2, \Pi_{0}$ es un vector $(2 \times 1)$ de términos constantes, $\Pi_{j}$ es una matriz $(2 \times n)$ de coeficientes autorregresivos con $j=$ $1,2, \cdots, p$ y $p$ es el número de rezagos, $\Omega_{i}$ es una matriz $(2 \times 2)$ de coeficientes de variables exógenas, y $\varepsilon_{t}$ es un vector $(2 \times 1)$ de innovaciones white noise.

En este modelo el vector contiene series estacionarias. Por ello, antes de estimar el VAR las series fueron evaluadas para determinar si son o no estacionarias. Se aplicaron la prueba aumentada de Dicky y Fuller (ADF) y la prueba de Phillips-Perron. Dado que es común encontrar que las series sean estacionarias en primeras diferencias, lo más conveniente es analizar la relación que existe entre ellas a través de la prueba de cointegración. La presencia de cointegración significa que dos o más variables integradas no estacionarias en niveles tienen una combinación lineal que es estacionaria $\sim I(0)$. Es decir, las variables no estacionarias no se alejan una de la otra y tienden a mantener una relación de largo plazo. Para conocer el número de vectores de cointegración $(r)$ en un sistema multivariado, se aplica el método de Johansen (1988) y Johansen y Juselius (1990). Esta técnica aplica máxima verosimilitud a un vector autoregresivo (VAR) bajo el supuesto que los errores tienen ruido blanco (white noise). El procedimiento se basa en dos estadísticos de prueba el estadístico trace y el estadístico maximum eigenvalue:

$$
\begin{gathered}
\text { Trace }=-N \sum_{i=r+1}^{m} \ln \left(1-\hat{\lambda}_{i}\right) \\
\lambda_{\max }=-N \ln \left(1-\hat{\lambda}_{r+1}\right)
\end{gathered}
$$

donde $N$ es el número de observaciones, $m$ es el número de variables endógenas y $\hat{\lambda}$ es el i-esimo eigenvalue estimado del polinomio característico. El estadístico de prueba trace establece la hipótesis nula de que hay al menos $r$ vectores de cointegración, mientras que la alternativa establece que hay más de $r$ vectores. 
La prueba del estadístico eigenvalue establece la hipótesis nula de que hay $r$ vectores de cointegración versus la alternativa de que hay $r+1$ vector.

Los datos provienen de diferentes fuentes. Las exportaciones totales y la inversión extranjera directa son publicadas por el Banco de México de manera mensual en millones de dólares. Por otro lado, el tipo de cambio renminbi-dólar estadounidense se obtuvo de la base de datos de la Reserva Federal de Estados Unidos y el índice de producción industrial de EUA base 2010 se obtuvo del INEGI con base en reportes de la OCDE. El periodo de análisis abarca 1995 a 2015 en frecuencia trimestral.

\section{Resultados}

La aplicación de las pruebas ADF y Phillips-Perron a la inversión extranjera directa $(I E D)$, exportaciones $(E X)$, índice de producción industrial de EUA $(I P U S)$ y tipo de cambio $(Y U A N)$ se realizó para determinar si las series tienen raíz unitaria y en su caso, conocer el orden de integración. Los resultados se presentan en el cuadro 2 e indican que las variables son estacionarias en primeras diferencias, sólo IED resultó estacionaria en niveles por lo cual se tiene la opción de utilizarla así o en primeras diferencias.

Cuadro 2. Resultado de la pruebas de raíz unitaria para determinar el orden de integración. Hipótesis nula de raíz unitaria.

\begin{tabular}{|c|c|c|c|}
\hline & $\begin{array}{r}\text { Prueba } \\
\text { Dickey-Fuller } \\
\text { aumentada }\end{array}$ & $\begin{array}{r}\text { Prueba } \\
\text { Phillips- } \\
\text { Perron }\end{array}$ & $\begin{array}{r}\text { Orden de } \\
\text { integración }\end{array}$ \\
\hline $\begin{array}{l}\text { Variable en } \\
\text { niveles y } \\
\text { primeras } \\
\text { diferencias }(\Delta)\end{array}$ & $\begin{array}{r}\text { Constante y } \\
\text { tendencia }\end{array}$ & $\begin{array}{r}\text { Constante y } \\
\text { tendencia }\end{array}$ & \\
\hline$I E D$ & $\begin{array}{r}-6.831 \\
{[0.000]}\end{array}$ & $\begin{array}{r}-6.914 \\
{[0.000]}\end{array}$ & $\mathrm{I}(0)$ \\
\hline$E X$ & $\begin{array}{r}-2.677 \\
{[0.248]}\end{array}$ & $\begin{array}{r}-3.009 \\
{[0.136]}\end{array}$ & \\
\hline YUAN & $\begin{array}{r}-1.822 \\
{[0.684]}\end{array}$ & $\begin{array}{r}-1.588 \\
{[0.789]}\end{array}$ & \\
\hline IPUS & $\begin{array}{r}-3.522 \\
{[0.043]}\end{array}$ & $\begin{array}{r}-2.382 \\
{[0.386]}\end{array}$ & \\
\hline$\triangle E D$ & $\begin{array}{r}-12.769 \\
{[0.000]}\end{array}$ & $\begin{array}{c}-52.308 \\
{[0.000]}\end{array}$ & $\mathrm{I}(1)$ \\
\hline $\begin{array}{l}\triangle E X \\
\triangle Y U A N\end{array}$ & $\begin{array}{r}-5.005 \\
{[0.000]} \\
-4.661 \\
{[0.002]}\end{array}$ & $\begin{array}{r}-12.020 \\
{[0.000]} \\
-4.752 \\
{[0.001]}\end{array}$ & $\mathrm{I}(1)$ \\
\hline$\triangle I P U S$ & $\begin{array}{r}-4.177 \\
{[0.007]}\end{array}$ & $\begin{array}{r}-3.404 \\
{[0.057]}\end{array}$ & $\mathrm{I}(1)$ \\
\hline
\end{tabular}

Nota: los valores en corchetes muestran las probabilidades..

Dado que las dos variables consideradas como endógenas, $E X$ e $I E D$, son I(1) es necesario aplicar la prueba de cointegración de Johansen y Juselius (1990) 


\section{Nueva Época REMEF (The Mexican Journal of Economics and Finance)}

para determinar si existe o no una relación de largo plazo, pues si es así, el modelo (5) debe estimarse, no como $V A R$, sino como un Vector con Corrección de Error (VEC por sus siglas en inglés). La prueba de cointegración requiere estimar un $V A R$ en niveles con las variables endógenas solamente, en este caso $E X$ e $I E D$. Después de calibrar varios vectores autoregresivos con diferente número de rezagos, los criterios de información Hannan-Quinn y $L R$ secuencial recomendaron la selección de siete rezagos. Una vez estimado el $\operatorname{VAR}(7)$ se comprobó que pasara las pruebas de diagnóstico a los residuales (ver anexo AI). El cuadro 3 contiene los resultados.

Cuadro 3: Prueba de cointegración Johansen - Juselius Intervalo: 1 a 6 rezagos

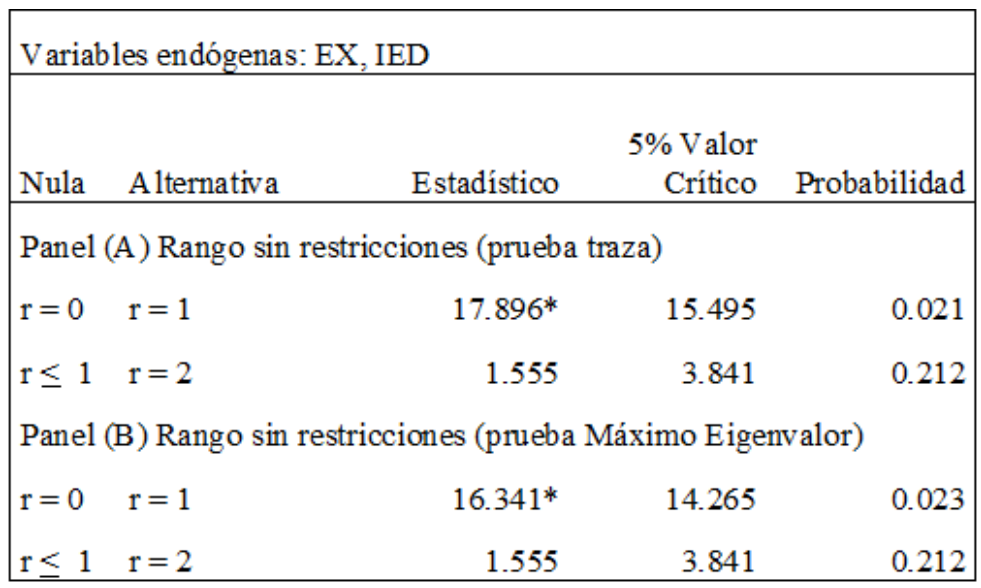

*: Denota el rechazo de la hipótesis nula de no cointegración a un nivel de 5 por ciento de significancia.

$r$ : indica el número de vectores de cointegración.

Los resultados indican el rechazo de la hipótesis nula de no cointegración, tanto el estadístico traza como el de máximo eigenvalor revelan que existe un vector cointegrante. Es decir, las exportaciones y la IED mantienen una relación de largo plazo, lo que implica que es necesario considerar un mecanismo de corrección de error en la especificación del modelo (5). Para ello, se requiere que la estimación de VEC, ya que así se permite que el comportamiento de largo plazo de las variables converja a una relación de equilibrio y se capture también el comportamiento a corto plazo. Dado que la mayoría de las variables son estacionarias en primeras diferencias, el modelo a estimar fue el siguiente:

$$
d y_{t}=\Theta y_{t-1}+\Psi_{1} \mathrm{~d} y_{t-1}+\ldots+\Psi_{p-1} \mathrm{~d} y_{t-p+1}+\Phi_{1} d X_{1 t}+\Phi d X_{2 t}+\varepsilon_{t}
$$

donde:

$$
d y_{t}=\left[\begin{array}{c}
d E X_{t} \\
d I E D_{t}
\end{array}\right] \Theta_{m}=\left[\begin{array}{cc}
\Theta^{d E X} & 0 \\
0 & \Theta^{d I E D}
\end{array}\right]
$$




$$
\begin{gathered}
y_{t-1}=\left[\begin{array}{c}
d E X_{t-1} \\
d I E D_{t-1}
\end{array}\right] \Psi_{i}=\left[\begin{array}{cccc}
\Psi_{11} & \Psi_{12} & \ldots & \Psi_{1, p-1} \\
\Psi_{21} & \Psi_{22} & \ldots & \Psi_{2, p-1}
\end{array}\right] \\
\mathrm{d} y_{t-j}=\left[\begin{array}{c}
\mathrm{d} y_{t-1} \\
\mathrm{~d} y_{t-2} \\
\cdots \\
\mathrm{d} y_{t-p+1}
\end{array}\right] \Phi_{k}=\left[\begin{array}{cc}
\Phi_{11} & \Phi_{12} \\
\Phi_{21} & \Phi_{22}
\end{array}\right] \\
d X_{t}=\begin{array}{c}
d Y U A N_{t} \\
d I P U S_{t}
\end{array} \varepsilon_{t}=\left[\begin{array}{c}
\varepsilon_{t}^{d E X} \\
\varepsilon_{t}^{d I E D}
\end{array}\right]
\end{gathered}
$$

$d y_{t}$ es un vector $(2 \times 1)$ de variables endógenas expresadas en su primera diferencia, $\Theta_{m}$ es una matriz diagonal de coeficientes $(2 \times 2), y_{(t-1)}$ es un vector de ecuaciones de cointegración, $\Psi_{i}$ es una matriz de coeficientes de las variables endógenas en su primera diferencia rezagadas, $\Phi_{k}$ es un vector de coeficientes $(2 \times 2)$ de las variables exógenas en sus primera diferencias, y $\varepsilon_{t}$ es un vector $(2 \times 1)$ de errores.

Para la selección de rezagos del modelo (8) se tomaron en cuenta los criterios de selección óptimos y después se aplicaron pruebas a los residuales para garantizar que el VEC(p) seleccionado no tuviera problemas de correlación serial, heterocedasticidad y estabilidad matemática. De esta forma, se estimó un VEC con seis rezagos, los resultados de la estimación completa se encuentran en el anexo A2. En el cuadro 4 sólo se presenta un resumen de las estimaciones. El panel a) contiene la estimación de los coeficientes del VEC(6) sólo con la constante, mientras que el panel b) contiene la estimación del VEC(6) con la inclusión de las variables exógenas $Y U A N$ e IPUS.

En general, el coeficiente de determinación (R cuadrada) del modelo mejora con la inclusión de las variables exógenas (de 0.565 a 0.709). El coeficiente del término de corrección de error de la ecuación DEX tiene el signo correcto, es significativo a $1 \%$ de probabilidad estadística e indica una velocidad de convergencia al equilibrio de $1.5 \%$. El término de corrección de error de la ecuación DIED aunque tiene el signo correcto no es estadísticamente significativo sin embargo, ambos términos indican que cualquier disturbio ocasionará que el sistema se corrija y vuelva al equilibrio.

\begin{tabular}{|c|c|c|c|}
\hline a) & $\operatorname{VEC}(6)$ & b) & $\operatorname{VEC}(6)$ \\
\hline \multicolumn{2}{|c|}{ Ecuación de cointegración } & \multicolumn{2}{|c|}{ Ecuación de cointegración } \\
\hline $\mathrm{EX}(-1)$ & 1 & $\mathrm{EX}(-1)$ & 1 \\
\hline \multirow[t]{2}{*}{$\operatorname{IED}(-1)$} & $6.560^{*}$ & $\operatorname{IED}(-1)$ & $6.043^{*}$ \\
\hline & [3.551] & & [ 2.532] \\
\hline c & -62.048 & $\mathrm{c}$ & -58.006 \\
\hline
\end{tabular}

Cuadro 4. Resumen de las estimaciones del vector de corrección de error con 6 rezagos. 
Cuadro 4. Resumen de las estimaciones del vector de corrección de error con 6 rezagos. (continuación)

\begin{tabular}{|lrr|lrr|}
\hline & DEX & DIED & & DEX & DIED \\
\hline Corrección de error & $-0.015^{*}$ & -0.050 & Corrección de error & $-0.010^{*}$ & -0.060 \\
& {$[-3.474]$} & {$[-1.519]$} & & {$[-2.319]$} & {$[-1.439]$} \\
C & $0.031^{*}$ & -0.070 & C & $0.018^{*}$ & -0.052 \\
& {$[3.610]$} & {$[-1.054]$} & & {$[2.435]$} & {$[-0.734]$} \\
& & & DYUAN & $-2.019 *$ & 3.473 \\
& & & & {$[-3.037]$} & {$[0.554]$} \\
& & & DIPUS & $2.419 * 4$ & -3.445 \\
& & & & {$[4.743]$} & {$[-0.716]$} \\
R cuadrada & 0.565 & 0.544 & R cuadrada & 0.709 & 0.550 \\
R.2 ajustada & 0.475 & 0.450 & R2 ajustada & 0.637 & 0.439 \\
Suma residua les2 & 0.186 & 11.188 & Suma residuales2 & 0.124 & 11.060 \\
S.E. ecuación & 0.054 & 0.421 & SE. ecuación & 0.045 & 0.426 \\
Estadistico F & 6.294 & 5.792 & Estadistico F & 9.892 & 4.964 \\
\hline & Estadistico & Probabilidad & & Estadistico & Probabilidad \\
LM test & 5.8 & 0.214 & LM test & 6.907 & 0.141 \\
White test & 79.521 & 0.431 & White test & 106.035 & 0.119 \\
Jarque Bera test & 49.281 & 0.000 & Jarque Bera test & 2.27 & 0.686 \\
Estabilidad matemática: el VEC impone una raiz & Estabilidad matemática: e1 VEC impone una raí \\
\hline
\end{tabular}

Nota: La selección de los rezagos óptimos empezó por considerar los criterios de selección propuestos por Akaike, Schwarz y Hannan-Quinn (Lütkepohl, 1991). A partir de ello, se aplicaron pruebas a los residuales del VEC empezando con el menor número de rezagos sugeridos hasta el mayor número que pasará las pruebas LM de correlación serial, White de heteroscedasticidad y de estabilidad matemática. El VEC con seis rezagos satisfizo estas condiciones. Nivel de significancia estadística igual a $1 \%$ y $*$ nivel de significancia estadística de $5 \%$.

Sobresale el hecho de que -para la ecuación que explica el crecimiento de las exportaciones DEX- el coeficiente que mide el efecto de las fluctuaciones del renminbi resultó estadísticamente significativo y además tiene el signo esperado (negativo). Esto significa que una depreciación del renminbi frente al dólar disminuirá la tasa de las exportaciones mexicanas en $2.02 \%$. Por lo tanto, las acciones de política monetaria que mantengan al renminbi depreciado contra el dólar, tendrán efectos negativos en las exportaciones mexicanas que compiten con productos similares en EUA. El coeficiente hallado es ligeramente superior al obtenido por Cuevas (2014) quien sólo midió el efecto de una depreciación sobre las exportaciones manufactureras, y obtuvo un coeficiente igual a $1.08 \%$. Comparando el mayor impacto de una depreciación del renminbi sobre las exportaciones totales versus las exportaciones manufactureras, podríamos decir que las manufacturas, al tener mayor especialización y contar con ventajas que no sólo dependen de los precios, presentan una menor sensibilidad a las fluctuaciones del renminbi. El signo negativo del coeficiente DYUAN también 
implica que por cada punto porcentual que se aprecie el renminbi, el crecimiento de las exportaciones mexicanas aumentará $2.02 \%$.

La evidencia indica que el coeficiente que mide la actividad económica industrial en EUA (DIPUS) y que directamente afecta la demanda de exportaciones mexicanas fue igual a 2.419, estadísticamente significativo y con el signo correcto. Por otro lado, las estimaciones indican que no hay relación entre las fluctuaciones renminbi y el crecimiento de la IED en México. Este hallazgo confirma en cierta medida la postura de Watkins (2015) en el sentido de que a pesar de que China ha ganado posición por sus precios bajos en el mercado de EUA, en términos de relaciones empresariales e inversión directa, la cercanía de EUA con México es un elemento que China no puede superar.

\section{Conclusiones}

La economía china desplazó de manera espectacular a México como principal socio comercial de los Estados Unidos de América. Esto se explica por el crecimiento exponencial de las exportaciones chinas hacia el mundo y hacia la economía estadounidense, determinado por factores tales como: su política cambiaria, sus ventajas comparativas y la alta productividad del trabajo (calificado y no calificado).

Por otra parte, a pesar que México ha perdido competitividad en algunos productos en los cuales mantuvo por mucho tiempo una participación importante en las importaciones del mercado estadounidense, tales como los productos textiles, la ropa, el calzado y aparatos de televisión, también es cierto que ha ganado participación en productos de exportación con mayor contenido de tecnología.

Considerando el total de las exportaciones mexicanas, éstas indican que los cambios en el valor del renminbi frente al dólar tienen un efecto estadísticamente significativo. El signo indica que una depreciación de la moneda reducirá la tasa de crecimiento de las exportaciones mexicanas a EUA en $2.019 \%$. Por el contrario, una apreciación del yuan aumentará la variación de las exportaciones. Al ser competidores cercanos, para México la fluctuación del renminbi representa un aspecto sobre el cual no tiene control y, por lo tanto, requiere buscar mecanismos que permitan incrementar la participación de productos donde se tiene ventaja basada, no sólo en precios, sino en contenido tecnológico, tiempos de entrega, calidad, etc. Desde el punto de vista de la IED, no se encontraron efectos significativos en los coeficientes, es decir, los flujos de IED a México parecen ser ajenos al valor de la moneda china. Quizás la cercanía geográfica con EUA sigue siendo un punto a favor para México.

En un espectro más amplio para contribuir a contrarrestar la competencia con China el gobierno mexicano debería impulsar una política cambiaria de largo aliento que promueva la competitividad de las exportaciones mexicanas. Para mejorar la competitividad del sector manufacturero mexicano, es necesario también que el gobierno fomente políticas industriales que mejoren la productividad del sector (pero en actividades clave) y promuevan la formación del trabajo calificado. 
38 Nueva Época REMEF (The Mexican Journal of Economics and Finance)

\section{ANEXOS}

A1. Estimación del $\operatorname{VAR}(7)$ para la prueba de cointegración.

\begin{tabular}{|c|c|c|}
\hline \multicolumn{3}{|c|}{$\begin{array}{l}\text { Muestra: 1996Q4 2015Q4 } \\
\text { No. observaciones: } 77 \text { después de ajustes } \\
\text { Estadístico t en [ ] }\end{array}$} \\
\hline & LN_EX & LN_IED \\
\hline LN_EX(-1) & $\begin{array}{r}1.061 \\
{[8.728]}\end{array}$ & $\begin{array}{r}0.352 \\
{[0.374]}\end{array}$ \\
\hline LN_EX(-2) & $\begin{array}{r}-0.183 \\
{[-1.046]}\end{array}$ & $\begin{array}{r}-0.436 \\
{[-0.323]}\end{array}$ \\
\hline LN_EX(-3) & $\begin{array}{r}-0.379 \\
{[-2.455]}\end{array}$ & $\begin{array}{r}1.853 \\
{[1.555]}\end{array}$ \\
\hline LN_EX(-4) & $\begin{array}{r}0.766 \\
{[5.542]}\end{array}$ & $\begin{array}{r}-0.859 \\
{[-0.805]}\end{array}$ \\
\hline LN_EX(-5) & $\begin{array}{c}-0.695 \\
{[-4.208]}\end{array}$ & $\begin{array}{r}-1.456 \\
{[-1.142]}\end{array}$ \\
\hline LN_EX(-6) & $\begin{array}{r}0.215 \\
{[1.15920]}\end{array}$ & $\begin{array}{r}3.070 \\
{[2.141]}\end{array}$ \\
\hline LN_EX(-7) & $\begin{array}{r}0.192 \\
{[1.51642]}\end{array}$ & $\begin{array}{r}-2.444 \\
{[-2.506]}\end{array}$ \\
\hline LN_IED(-1) & $\begin{array}{r}-0.014 \\
{[-0.85688]}\end{array}$ & $\begin{array}{r}0.281 \\
{[2.30760]}\end{array}$ \\
\hline LN_IED(-2) & $\begin{array}{r}0.008 \\
{[0.49037]}\end{array}$ & $\begin{array}{r}-0.246 \\
{[-1.88615]}\end{array}$ \\
\hline LN_IED(-3) & $\begin{array}{r}-0.024 \\
{[-1.40838]}\end{array}$ & $\begin{array}{r}0.379 \\
{[2.93121]}\end{array}$ \\
\hline LN_IED(-4) & $\begin{array}{r}0.000 \\
{[-0.00926]}\end{array}$ & $\begin{array}{r}-0.082 \\
{[-0.57320]}\end{array}$ \\
\hline LN_IED(-5) & $\begin{array}{r}-0.023 \\
{[-1.29215]}\end{array}$ & $\begin{array}{r}0.214 \\
{[1.57258]}\end{array}$ \\
\hline LN_IED(-6) & $\begin{array}{r}0.004 \\
{[0.25387]}\end{array}$ & $\begin{array}{r}-0.082 \\
{[-0.61785]}\end{array}$ \\
\hline LN_IED(-7) & $\begin{array}{r}-0.042 \\
{[-2.374]}\end{array}$ & $\begin{array}{r}0.106 \\
{[0.778]}\end{array}$ \\
\hline Constante & $\begin{array}{r}0.972 \\
{[3.54096]}\end{array}$ & $\begin{array}{r}2.450 \\
{[1.15662]}\end{array}$ \\
\hline R-cuadrada & 0.983 & 0.292 \\
\hline R-cuadrada ajustada & 0.980 & 0.132 \\
\hline Suma residuales2 & 0.185 & 10.999 \\
\hline S.E. ecuación & 0.055 & 0.421 \\
\hline F-estadístico & $\begin{array}{r}261.192 \\
\text { Estadístico }\end{array}$ & $\begin{array}{r}1.826 \\
\text { Probabilidad }\end{array}$ \\
\hline LM test & 3.773 & 0.437 \\
\hline White test & 77.519 & 0.677 \\
\hline Jarque Bera test & 50.836 & 0.000 \\
\hline
\end{tabular}


A2. Estimaciones del Vector de corrección de error. 6 rezagos.

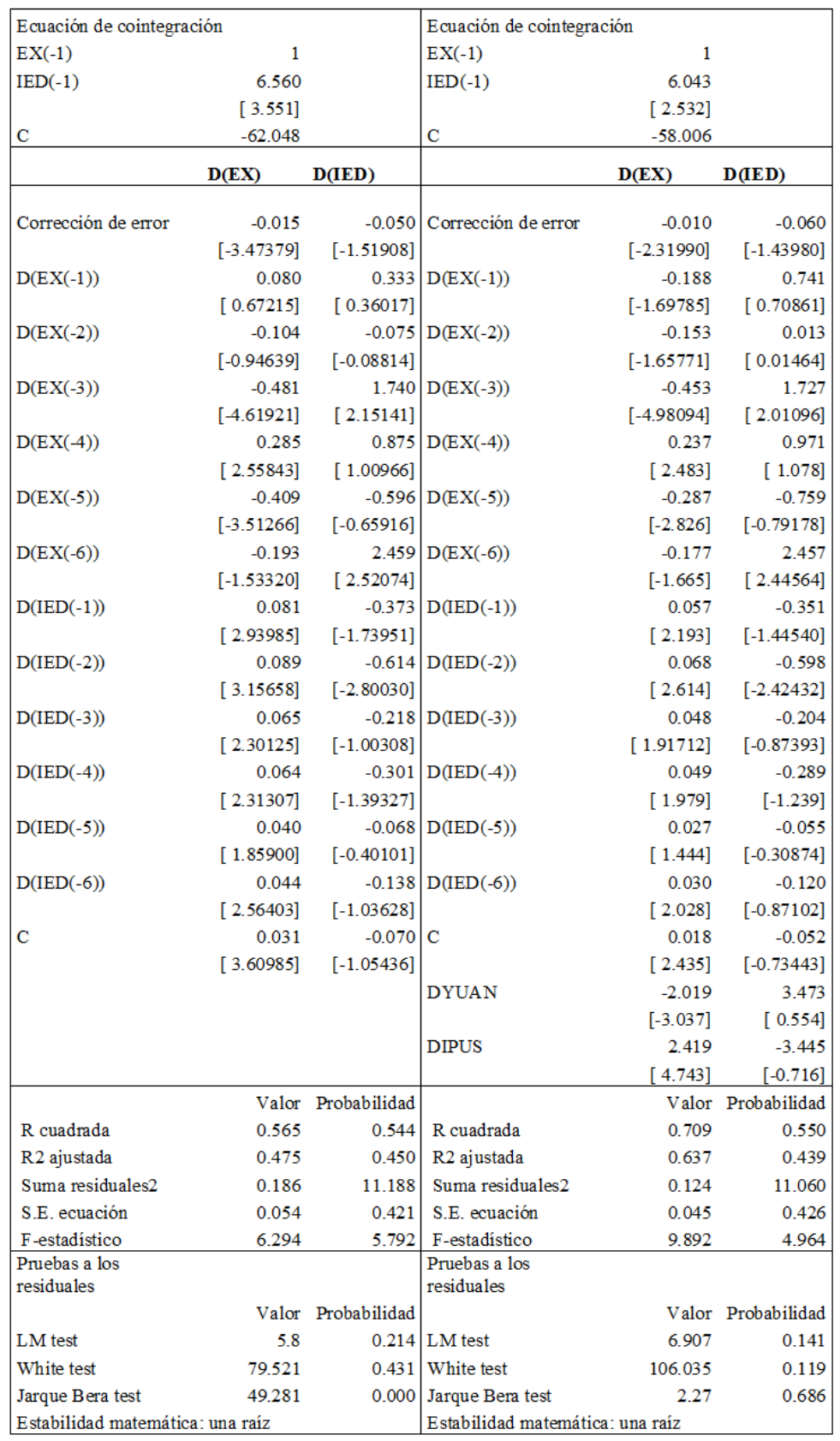




\section{Bibliografía}

Banxico. (2016). Banco de México Estadísticas. Consultado el 25 de mayo de 2016. Disponible en http://www.banxico.org

Bhagwati, J., Panagariya, A.,\& Srinivasan, T. (1998). Lectures on International Trade, 2 ed. England: MIT Press.

Carrillo, J., \& Plascencia, I. (2007). La industria de los televisores y la competencia México China por el mercado estadounidense. En Dussel, E., Trapaga, Y. (Ed.), China y México implicaciones de una nueva relación. México nuestro tiempo. México: Nuestro Tiempo la Jornada, pp. 209-233.

Chávez, F., \& Marin, L. (2007). México y China: competencia en el mercado de Estados Unidos. Comercio Exterior, 57(11), 931-944.

Cline, W., \& Williamson, J. (2008). Estimates of the Equilibrium of the Exchange Rate of the Renminbi. En Goldstein, M., Lardy, E. (Ed.), Debating Chinas Exchange Rate. Washington: Peterson Institute for International Economics, pp. 131-154.

Comtrade. (2016). UN Comtrade database. Consultado el 8 de febrero de 2016. Disponible en http://comtrade.un.org/

Corden, M. (2009). Chinas Exchange Rate Policy, its Current Account Surplus and Global Imbalances. The Economic Journal, 119(541), 430-441.

Cuevas, V. (2016). Cost and Relative Price Competitiveness of the Mexican Manufacturing Industry in the U.S. Market. Frontera Norte, 28(55), 53-78.

Cuevas, V. (2014). La paridad yuan-dólar y su efecto en las exportaciones manufactureras mexicanas. Portes Revista Mexicana de estudios sobre la cuenca del pacífico, 8(enerojunio), $7-34$.

Dussel, E. (2009). The Mexican Case. En Jenkins, R., Dussel, E. (Ed.), China and Latin America. Alemania: German Development Institute, pp. 279-385.

Dussel, E., \& Gallagher, K. (2013). NAFTAs Uninvited Guest: China and the Desintegration of North America Trade. CEPAL Review, 83-108.

Federal Reserve System. (2016). Foreign Exchange Rates. Consultado el 11 de enero de 2016. Disponible en https://www.federalreserve.gov/releases/h10/hist/dat00_ch.htm

Frankel, J., \& Wei, S. (2007). Assessing China's Exchange Rate Regime. Economic Policy, $51,575-614$.

Goldstein, M., \& Lardy, N. (2008). Debating Chinas Exchange Rate Policy. Washington: Peterson Institute for International Economics.

Gracia-Hernández, M. (2013). Competencia entre México y China en el interior del mercado de Estados Unidos. Economía, Sociedad y Territorio, 13(1), 37-78.

Instituto Nacional de Estadística Geografía e Informática. (2016). Banco de Información Económica. Consultado el 11 de enero de 2016. Disponible en http://www.inegi.org.mx /sistemas/bie/default.aspx

International Trade Center. (2016). Trade Map, statistics for international business development. Consultado el 14 de mayo de 2016. Disponible en www.trademap.org/bilateral_ TS.aspx

Johansen, S. (1988). Statistical Analysis of Cointegrating Vectors. Journal of Economics Dynamics and Control, 12, 213-254.

Johansen, S., \& Juselius, K. (1990). Maximun Likelihood Estimation and Inference on Cointegration with Applications to the Demand for Money. Oxford Bulletin of Economics and Statistics, 52, 169-210.

Krugman, P. (1980). Scale Economies, Product Differentiation and the Pattern of Trade. American Economic Review, 70, 950-959.

Krugman, P., \& Obstfeld, M. (2006). Economía internacional, teoría y práctica, 7 ed. México: Pearson.

Lütkepohl, H. (1991). Introduction to Multiple Time Series Analysis. Nueva York: SpringerVerlag.

Mendoza, E. (2010). El comportamiento de la industria manufacturera de México ante la recesión económica de EUA. Revista de Economía, 27(75), 11-35. 
Organización Mundial del Comercio, OMC. (1994). Acuerdo relativo a la aplicación del artículo VI del acuerdo general sobre aranceles aduaneros y comercio de 1994. Consultado el 20 de 6 de 2016. Disponible en https://www.wto.org/spanish/docs_ s/legal_s/19-adp.pdf

Perrotini, I., \& Liu Sun, X. (2015). El papel del tipo de cambio y la política monetaria en el crecimiento económico de China y México. En Dussel, E. (Ed.), América Latina y el Caribe China. Economía Comercio e Inversión. México: Unión de Universidades de América Latina, pp. 35-48.

Shannon, K. (2010). Currency War: China Stands Firm on Yuan as Global Criticism Escalates. Consultado el 10 de diciembre de 2010. Disponible en http://moneymorning. com/2010/10/14/currency-war-9/

The Peoples Bank of China. (2015). Law of the People's Republic of China on The People's Bank of China. Consultado el 15 de agosto de 2016. Disponible en http://www.pbc.gov. cn/english/130733/2941519/2015082610501049304.pdf

Watkins, R. (2015). La competencia entre México y China en las importaciones de Estados Unidos (2000-2013). En Dussel, E. (Ed.), América Latina y el Caribe-China. Economía, Comercio e Inversión. México: UDUAL, pp. 225-248.

World Trade Organization, WTO. (2001). World Trade Organization. Consultado el 10 de junio de 2016. Disponible en https://www.wto.org/english/news_e/pres01_e/pr243_e. htm

Xiaochuan, Z. (2005). The Peoples Bank of China. Consultado el 25 de junio de 2016. Disponible en http://www.pbc.gov.cn/english/130724/2829809/index.html

US Census Bureau (2016). Foreign Trade. Consultado el 3 de enero de 2016. Disponible en https://www.census.gov/foreign-trade/balance/index.html 